\title{
Fuzzy Logic-based Virtual Cell Design in Ultra-Dense Networks
}

\author{
Qian Liu* (D), Gang Chuai, Weidong Gao and Kaisa Zhang
}

\begin{abstract}
One of the main trends in the fifth generation (5G) cellular network is the bifurcation of systems that enable network densification. With this trend, ultra-dense networks (UDN) become a pillar technology to provide high data rates gains. However, these gains come at the expense of more complex interference and higher handover frequency. The user-centric virtual cell conception has been proposed to solve these problems. But it still has encountered some challenges in practical application. In this paper, we propose a fuzzy logic-based virtual cell design to balance data rates and resource overhead in order to improve virtual cell applicability. In our design, we consider a practical UDN scenario with imperfect channel state information (CSI) feedback and model it by stochastic geometry theory. Then, we find the optimal radius of virtual cells by maximizing the system spectral efficiency. On the basis, we propose a fuzzy logic-based activation strategy selection (FASS) algorithm to rationally choose the virtual cell activation threshold and resources allocation scheme under different traffic loads and quality of service (QoS) requirement. Simulation results demonstrate that the proposed solution can effectively eliminate the interference, obviously improve the user's system spectral efficiency and dynamically adapt to different traffic loads.
\end{abstract}

Keywords: Fuzzy logic, Stochastic geometry, Virtual cell, UDN, 5 G

\section{Introduction}

The fifth generation (5G) wireless networks are required to satisfy the increasing user population and their traffic demands. The most substantial amount of this system performance gains will be obtained by means of network infrastructure densification [1]. Thus, UDN has been identified as one of pillar technologies in $5 \mathrm{G}$ to significantly improve system capacity and coverage [2]. In theoretical analysis, UDN can bring significant benefits in terms of proximal transmission. However, the densely deployed BSs and irregular network topology introduce a huge amount of problems. More complex interference and frequent handover will offset gains. To meet these challenges, the user-centric virtual cell concept has been proposed [3].

A user-centric virtual cell is configured with several cooperate BSs and a user. The user is located in the center and BSs in a circle area with radius D. Virtual cells can convert interference signals into useful

\footnotetext{
* Correspondence: LiuQian@bupt.edu.cn

Key Laboratory of Universal Wireless Communications, Ministry of Education, Beijing University of Posts and Telecommunications, Beijing 100876, People's Republic of China
}

signals and offer users seamless moving experience [4]. There have been quite a few studies in multiple BSs transmissions. Such as dynamic cell clustering [5-9], coordinated multipoint transmission (CoMP) [10-12], and virtual multiple-input multiple-output (MIMO) [13-15].

Dynamic cell clustering is widely used in many fields due to its low complexity. In [5], they proposed a service-aware user-centric clustering and scheduling scheme. With the proposed scheme, throughput improvements can be achieved for both center and edge users in a fair fashion. In [6], authors investigated an adaptive clustering for irregular topologies. Some papers handled resources allocation with clustering in different scenarios [7-9]. Coordinated multi-point (CoMP) transmission is considered as an efficient technique to improve cell-edge performance. In [10], authors compared performances of different CoMP schemes in downlink. In [11], a novel user-centric CoMP scheme was proposed to improve energy efficiency in dense heterogeneous network. In [12], a two-step joint clustering and scheduling scheme was proposed for heterogeneous 
networks. A large number of transmission nodes that configure a single antenna make up a distributed antenna system (DAS). If the DAS provides service for multiple users, it will be equivalent to a virtual MIMO system. In [13], an adaptive virtual MIMO technology was used to increase spectral efficiency. In [14], authors develop a new formulation of the beamforming problem for sum-rate maximization in virtual cell networks and analyze the structure of its optimal solutions. In [15], a virtual MIMO broadcasting transceiver has been proposed to reduce the computational complexity and improve the end-toend sum rate.

However, it was found that accurate CSI feedback and tight BS synchronization required for coherent cooperation may limit the achievable gains of above technologies [16]. Techniques with less stringent requirement will be first choice for virtual cell formation. In this article, we adopt non-coherent joint transmission (NCJT) technique to support virtual cell formation. In NCJT, a user's signal is transmitted by multiple cooperating BSs without prior phasemismatch correction and tight synchronization across BSs. At the user, the received signals are noncoherently combined, thereby providing opportunistic power gains [17].

Although NCJT has so many advantages, it still has an application restriction. With aggressive cooperation, more BSs will be allowed to participate in joint transmission and resource overhead will increase faster. NCJT may be favorable only to a lightly loads extent since the additional load imposed on BSs eventually outpaces the spectral efficiency gains. Thus, NCJT is considered more suitable for deployment in lightly loaded scenario [17]. It will greatly limit the deployment of virtual cells in a practical scenario. In order to break this restriction, the effect of different loads should be taken into account in virtual cell design. To the best of our knowledge, there is no virtual cell design to simultaneously consider the effect of imperfect CSI and traffic loads. In this paper, we propose a novel fuzzy-based virtual cell design to balance data rates and practical resource overhead by flexible activation strategies.

The main contributions of this paper are as follows:

(1) In our design, we consider deploying virtual cells in a typical ultra-dense network with imperfect CSI feedback. We model the impact of imperfect CSI as a new interference by stochastic geometry theory.

(2) We investigate the virtual cell optimal radius to maximize the system spectral efficiency.

(3) We propose a fuzzy logic-based activation strategy selection (FASS) algorithm for virtual cells. Users can achieve a strong applicability for different loads by our virtual cell design.

The rest of this paper is organized as follows. In Section II, we provide the system model and assumptions. Section III, the virtual cell optimal radius is investigated. On this basis, we propose a FASS algorithm to find the optimal activation strategy in Section IV. We validate the proposed design and discuss simulation results in Section V. Finally, Section VI concludes the paper and outlines our future work.

\section{System model}

There are many different definitions of UDN in literatures [18-20]. In our paper, we consider a practical scenario of UDN. The denser employment of BSs makes network exhibit random topologies. We use stochastic geometry tools to capture this network characteristic [21]. For the influence of imperfect CSI, we consider it as a new interference resource. In addition, we install a network controller unit (NCU) by splitting the control plane (C-plan) and user plan (U-plan) to harvest densification gains [22]. This unit takes charge of virtual cell formation, wireless radio resources allocation, and mobility management. BSs only undertake the task of data transmission.

We model BSs and mobile users with stationary Poisson point process (PPP). The BSs are modeled as a PPP $\Phi_{\mathrm{BS}}$ with density $\lambda_{\mathrm{BS}}$. They are assumed to be equipped with a single antenna of equal transmit power. The single-antenna mobile users are spatially distributed according to an independent PPP $\Phi_{\mathrm{U}}$ with density $\lambda_{\mathrm{U}}$. Let $h_{i j}=\sqrt{l_{i j}} f_{i j}$ represents the channel gain for the user $j$ from the BS $i . l_{i j}=\left|x_{i}-x_{j}\right|^{-\alpha}$ represents the corresponding path loss. $x_{i}$ and $x_{j}$ denotes the random location of the BS $i$ and the user $j$, respectively. $\alpha>2$ is the path loss exponent. Assuming a Rayleigh fading environment, $\left\{f_{i j}\right\}$ are independently and identically distributed (i.i.d) complex Gaussian random variables with zero mean and unit variance. Each channel is estimated independently. The composite channel of a virtual cell subsumes all cooperative channels.

The user-centric virtual cell is a circular region around the user with radius $D$. The set of BSs within virtual cell region of user $j$ denoted as $V_{j} \triangleq\left\{\mathrm{BS}_{i} \in \Phi_{\mathrm{BS}}, \mid\right.$ $\left.x_{\mathrm{BS}_{i}}-x_{j} \mid \leq D\right\}$. Information cannot be sent reliably over a link that in a deep fading. If BSs participating in joint transmissions, their RSPRs have to greater than or equal to a threshold $T . a_{V_{j}}^{\mathrm{ia}_{x}} \in\{0,1\}$ is the activation indictor for each BS. $a_{V_{j}}^{\mathrm{ia}_{x}}=0$ is inactive and $a_{V_{j}}^{\mathrm{ia}_{x}}=1$ is active. In addition, $\mathrm{ia}_{x}$ is an indicator of resources 
allocation scheme. $\mathrm{ia}_{1}$ is "silent" scheme, which indicates resources allocated for NCJT cannot be reused by other inactive $\mathrm{BSs}$ in a virtual cell. $\mathrm{ia}_{2}$ is "reuse" scheme, which indicates resources allocated for NCJT can be reused by other inactive BSs. All active BSs in a virtual cell provide a non-coherent joint transmission for the user. The user served by its own virtual cell as shown in Fig. 1.

In our paper, the channel estimation error according to imperfect CSI is equivalent to a new interference resource. Therefore, the aggregate interference mainly includes three categories: (1) sum interference caused by inactive BSs in virtual cells (resources allocation scheme is reuse); (2) sum interference created by BSs outside virtual cells; (3) residual interference due to imperfect CSI.

$S_{j}$ denotes the transmitted signal to user $j, n_{j}$ is the corresponding complex Gaussian noise with zero mean and variance $\sigma_{n}^{2}$. The received signal at user $j$ is given by

$$
\begin{aligned}
y_{j} & =\sum_{i \in \Phi_{\mathrm{BS} \cap V_{j}}} \sqrt{\left(1-\sigma_{\mathrm{MMSE}, i j}\right)^{2}} h_{i j} S_{j} a_{V_{j}}^{\mathrm{i} \mathrm{a}_{x}}+\sum_{i \in \Phi_{\mathrm{BS} \cap V_{j}}} h_{i j} S_{j}\left(1-a_{V_{j}}^{\mathrm{i} a_{x}}\right) \\
& +\sum_{i \in \Phi_{\mathrm{BS}} \cap \overline{V_{j}}} h_{i j} S_{j}+\sum_{i \in \Phi_{\mathrm{BS}} \cap V_{j}} \sigma_{\mathrm{MMSE}, i j} h_{i j} S_{j} a_{V_{j}}^{\mathrm{i}_{\mathrm{x}}}+n_{j}
\end{aligned}
$$

Where, $\sigma_{\mathrm{MMSE}}, \mathrm{ij}^{2}$ is the minimum mean-square error of ,$i$-th channel estimate given $K$ transmitters for $j$-th user. For pilot-based channel estimation, $\sigma_{\mathrm{MMSE}},{ }_{i j}^{2}$ has the similar form with [23].

$$
\sigma_{\mathrm{MMSE}, i j}^{2}=\frac{1}{1+E_{H i j}\left[\operatorname{SINR}_{\text {pilot }, i j}\right] \frac{N_{\text {pilot }}}{K}}
$$

$K$ is the number of active BSs in a virtual cell. $\eta$ is the signal-to-noise ratio. $N_{\text {pilot }}$ is the total number of pilot resource blocks dedicated for channel state estimation. Assuming these resources share among active BSs and the inter-reference signals interference is avoided in a virtual cell. $\sigma_{\text {MMSE, }}{ }^{2}$ is further expressed as

$$
\sigma_{\mathrm{MMSE}, i j}{ }^{2}=\frac{1}{1+\frac{\left|h_{i j}\right|^{2}}{\sum_{i \in \Phi_{\mathrm{BS}} \cap \bar{V}_{j}}\left|h_{i j}\right|^{2}+1 / \eta \frac{N_{\text {pilot }}}{K}}}
$$

The signal to interference plus noise ratio (SINR) at the user $j$ can be expressed as

$$
\mathrm{SINR}=\frac{R}{I_{c}+I_{\bar{c}}+I_{\mathrm{CSI}}+{ }^{1} / \eta}
$$

Where

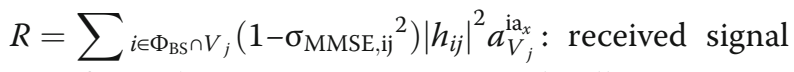
power from the active BSs in a virtual cell.

$$
I_{c}=\sum_{i \in \Phi_{\mathrm{BS}} \cap V_{j}}\left|h_{i j}\right|^{2}\left(1-a_{V_{j}}^{\mathrm{ia}_{2}}\right): \text { sum interference }
$$
caused by inactive BSs in a virtual cell. Especially, when resource allocated indictor is $\mathrm{ia}_{1}$ or $T=0, I_{c}=0$.

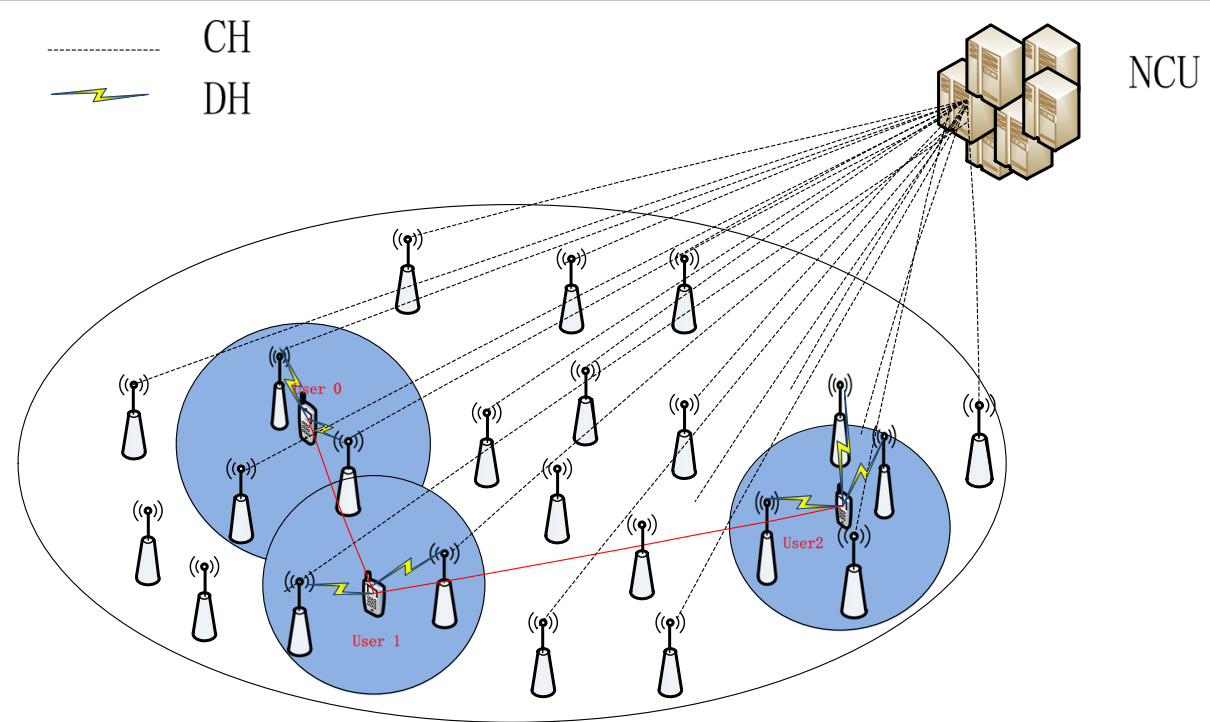

Fig. 1 Virtual cell architecture 
$I_{\bar{c}}=\sum_{i \in \Phi_{\mathrm{BS} \cap} \overline{V_{j}}}\left|h_{i j}\right|^{2}$ : sum interference created by BSs outside a virtual cell.

$I_{\mathrm{CSI}}=\left.\sum_{i \in \Phi_{\mathrm{BS} \cap V_{j}}} \sigma_{\mathrm{MMSE}, i j}{ }^{2} h_{i j}\right|^{2} a_{V_{j}}^{\mathrm{ia}_{x}}:$ residual interference due to imperfect CSI.

$\eta$ : signal-to-noise ratio.

\section{The optimal radius of virtual cell}

\subsection{Signal power}

The received signal power $R$ is sum of multiple serving BSs channel gains. Considering the number of activate BSs in a virtual cell is $K$, the Laplace transform of the received signal power $R$ is given by

$$
\mathcal{L}_{R}(\mathrm{~s}) \triangleq E\left[e^{-s R}\right]=\left(\frac{2}{\alpha \mathrm{D}^{2}} \int_{D^{-\alpha}}^{\infty} t^{-1-\frac{2}{\alpha}}\left(1-e^{-\frac{T}{t}}+\frac{e^{-\frac{T}{t}(u+1)}}{u+1}\right) \mathrm{dt}\right)^{K}
$$

Where, $u=\frac{s t^{2}}{t+K /\left[N_{\text {pilot }}\left(E\left[\bar{I}_{\mathbf{C}}\right]\right)+1 / \eta\right]}$

Proof: see (21) in Appendix.

\subsection{Interference power}

For residual interference due to the estimation error of imperfect CSI, in loosely speaking, we treat it residual as an independent Gaussian noise [24]. In [25], authors showed that the Gamma distribution can provide a reasonably tight fit to the statistics of Poisson interference.
Thus, we adopt a Gamma random variable $\tilde{I}$ to approximate the aggregate interference. The variable $\tilde{I}$ with distribution as

$$
\mathbb{P}(\tilde{I} \leq z)=1-\Gamma(k, z / \theta) / \Gamma(k)
$$

Then, the variable $\tilde{I}$ can be computed using Campbell's theorem as

$$
\begin{aligned}
E[\tilde{I}]= & E\left[2 \pi \lambda \int_{0}^{D} f_{i j}{ }^{2} r^{-\alpha+1}\left(1-a_{V_{j}}^{\mathrm{i} \mathrm{a}_{2}}\right) d r\right]+2 \pi \lambda \int_{\mathrm{D}}^{\infty} E\left(f_{i j}{ }^{2}\right) r^{-\alpha+1} d r \\
+ & {\left[\left[\sum_{i=0}^{K-1} \sigma_{\mathrm{MMSE}, i j}{ }^{2} f_{i j}{ }^{2} r^{-\alpha}\left(1-a_{V_{j}}^{\mathrm{i} \mathrm{a}_{2}}\right)\right]+1 / \eta\right.} \\
\operatorname{Var}[\tilde{I}]= & E\left[2 \pi \lambda \int_{0}^{\mathrm{D}} f_{i j}{ }^{4} r^{-2 \alpha+1}\left(1-a_{V_{j}}^{\mathrm{i} \mathrm{a}_{2}}\right) d r\right]+2 \pi \lambda \int_{D}^{\infty} E\left(f_{i j}{ }^{4}\right) r^{-2 \alpha+1} d r \\
& +E\left[\sum_{i=0}^{K-1} \sigma_{\mathrm{MMSE}, i j}{ }^{4} f_{i j}^{4} r^{-2 \alpha}\left(1-a_{V_{j}}^{\mathrm{ia}_{x}}\right)\right]-E\left[\sum_{i=0}^{K-1} \sigma_{\mathrm{MMSE}, i j}{ }^{2} f_{i j}{ }^{2} r^{-\alpha}\left(1-a_{V_{j}}^{\mathrm{i} \mathrm{a}_{x}}\right)\right]
\end{aligned}
$$

The parameters $k$ and $\theta$ satisfy the relations $E[\tilde{I}]$ $=k \theta$ and $\operatorname{Var}[\tilde{I}]=k \theta^{2}$. With (7) and (8), we can solve the specific value for $k$ and $\theta$. The expressions are (22) and (23) in Appendix.

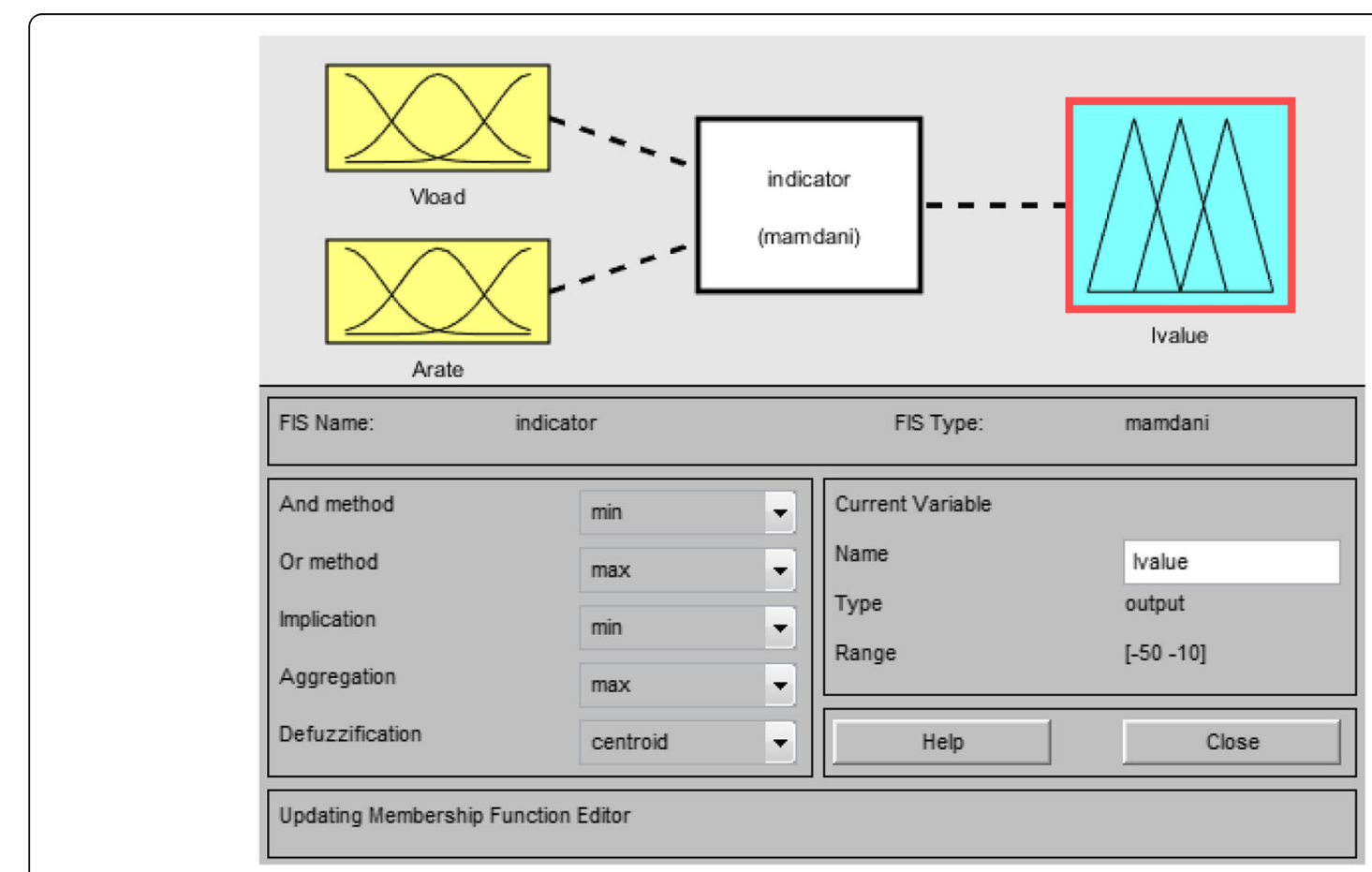

Fig. 2 Fuzzy logic system 

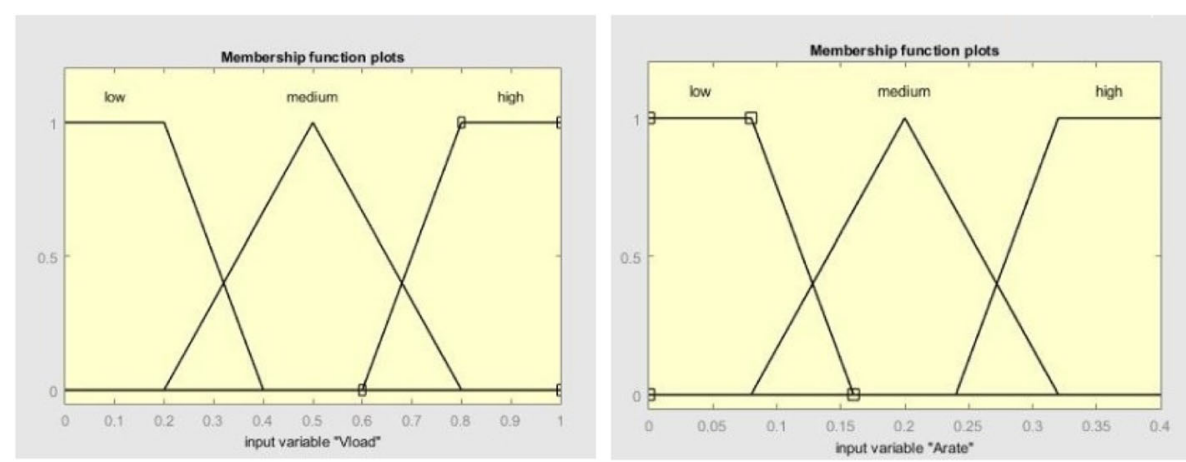

Fig. 3 Membership functions of inputs

\subsection{Optimal radius}

Base on the previous analysis, the approximation of $\mathrm{cu}-$ mulative distribution function (CDF) of SINR can be derived with the familiar form in ([26], Corollary1)

$$
\begin{aligned}
\mathbb{P}(\operatorname{SINR} \leq \beta) & \approx(k-k) \mathcal{L}_{\mathrm{R}}^{\mathrm{k}}\left(\frac{1}{\theta \beta}\right) \frac{(\theta \beta)^{-k}}{k !} \\
& +\sum_{m=0}^{k} \mathcal{L}_{R}^{(m)}\left(\frac{1}{\theta \beta}\right) \frac{(\theta \beta)^{-m}}{m !}
\end{aligned}
$$

The system spectral efficiency $C_{T, \mathrm{ia}_{\mathrm{x}}}$ can be obtained using the relation $R=\log _{2}(1+\mathrm{SINR})$ and $\mathrm{E}[\mathrm{R}]=\int_{0}^{\infty} \mathbb{P}($ $\left.\operatorname{SINR} \geq 2^{\mathrm{T}}-1\right) \mathrm{d} \tau$.

$$
C_{T, \mathrm{ia}_{x}}=E[R] \triangleq \frac{1}{\ln 2} \int_{\beta=0}^{\infty} \frac{1}{\beta+1}(1-\mathbb{P}(\operatorname{SINR} \leq \beta)) \mathrm{d} \beta
$$

From (4), we know that the signal power is an increasing function of virtual cell radius $D$. When radius $D$ is small, the system spectral efficiency is dominated by the signal power, which would be improved by virtual cell area expansion. We can also see that the mean of aggregate interference is an increasing function of the virtual cell radius $D$ from (7). When the virtual cell radius $D$ becomes large, the resident interference caused by pilot-based channel estimation keeps on increasing and becomes a main restriction on virtual cell size. The gain of the signal power gradually saturates, and it is finally overwhelmed by the increase of the aggregate interference power. Given $\lambda_{\mathrm{BS}}$ and $N_{\mathrm{pi}-}$ lot, it exists an optima virtual cell radius $D_{\text {best }}$ to maximize the system spectral efficiency.

$$
D_{\text {best }}=\arg \max C_{T, \mathrm{iax}_{x}}(D)
$$

\section{Fuzzy logic-based activation strategy selection algorithm}

In the previous section, we have discussed the optimal radius of virtual cell. Given $\lambda_{\mathrm{BS}}, T$, and $\mathrm{ia}_{x}$, the maximum system spectral efficiency can be obtained with $D_{\text {best }}$. In light-load scenario, we choose aggressively activation threshold $T$ and silent resources allocation scheme to maximize the rate performance. A

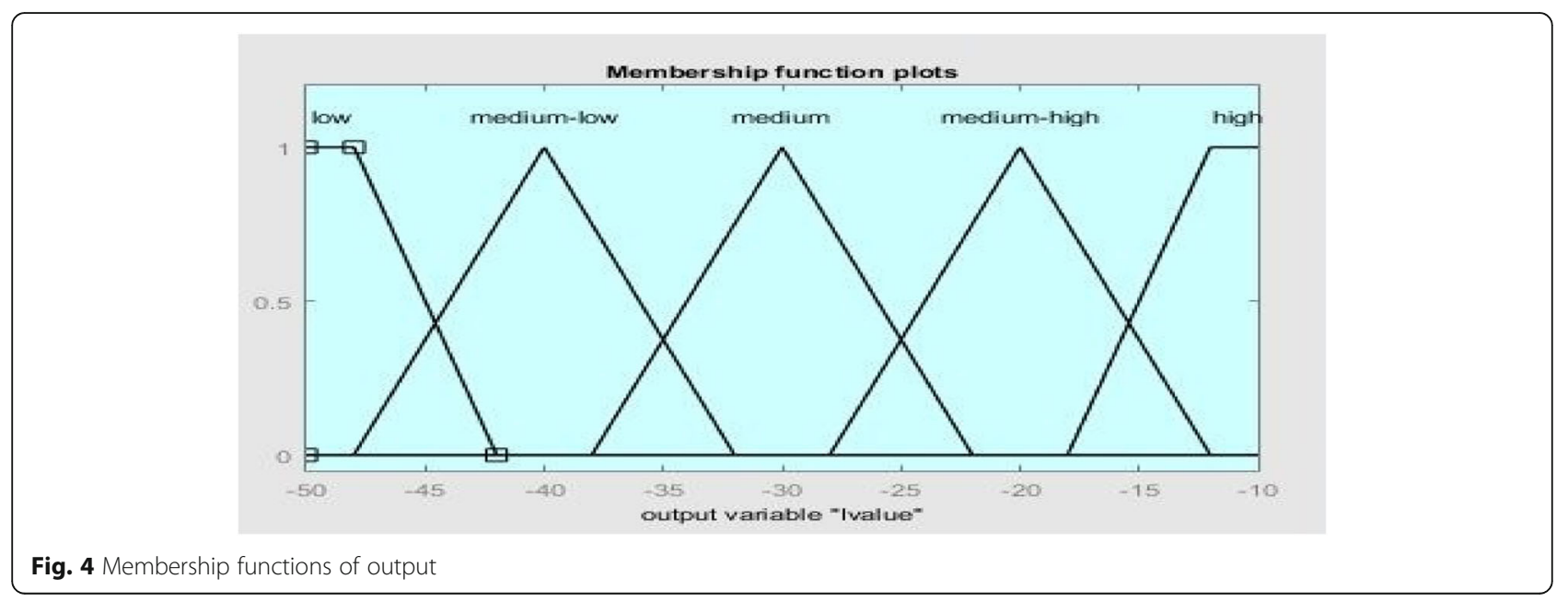


Table 1 Reasoning rules

\begin{tabular}{llll}
\hline Adjust & Load & & \\
\cline { 2 - 4 } rate & Low & Medium & High \\
\hline Low & Medium & Medium-high & High \\
Medium & Medium-low & Medium & Medium-high \\
High & Low & Medium-low & Medium \\
\hline
\end{tabular}

low threshold $T$ allows more BSs to participate in a joint transmission. Silent resources allocation scheme means that the resources allocated to NCJT cannot be reused by other inactive BSs in a virtual cell. It can make the interference $I_{c}=0$. However, these resources reserved for NCJT may become unacceptable when virtual cells in a high-load condition. The other users may possibly suffer from radio resources shortage. The importance of resource saving is higher than that of data rates at this time. We have to choose the reuse scheme, despite which increasing the interference within virtual cells. With the loads increasing continuously, the number of active BSs can be further decease by high activation thresholds $T$. We just protect users to obtain the guaranteed bit rates according to requirements of QoS.

From above analysis, we can see that data rate performance is tightly coupled with the traffic loads. But in a cooperative scenario, it is difficult to model and analyze due to different cross-cell scheduling [27, 28]. Instead of characterizing the impact of actual loads on network performance, we choose an alternative way to investigate this effect. It is capturing the preference for resources allocation scheme under different loads conditions.

To NCJT mechanism, there are two main resources allocation schemes: silent and reuse. (1) Silent, resources allocated to NCJT cannot be reused by inactive BSs to avoid intra-cell interference. Thereby, $I_{c}=0$. However, the loads on inactive BSs are increased. (2) Reuse, resources allocated to NCJT can be reused by inactive BSs. This scheme can improve the utilization of resources, but cause interference in a virtual cell. There is a tradeoff relationship between resources consumption and data rates. We can get the resources saving by switching resources allocation scheme from silent to reuse. It directly translates into a load reduction for virtual cells. The resources saving ration is as (12).

$$
\begin{gathered}
\Delta \text { Resource }=1-E\left[\frac{\sum_{i \in \Phi_{\mathrm{BS} \cap_{j}}} a_{V_{j}}^{\mathrm{ia}_{2}}}{\sum_{i \Phi_{\mathrm{BS} \cap V_{j}}} i}\right] \\
=1-E\left[\frac{E\left[\sum_{i \in \Phi_{\mathrm{BS} \cap V_{j}}} a_{V_{j}}^{\mathrm{ia}_{2}}\right]}{\mathrm{K}}\right] \\
=1-E\left[\min \left\{1,\left(\frac{\mathrm{TD}^{\alpha}}{f_{i j}{ }^{2}}\right)^{-\frac{2}{\alpha}}\right\}\right]
\end{gathered}
$$

In addition, we can adjust the number of active BSs by activation threshold $T$ so as to further reduce resource

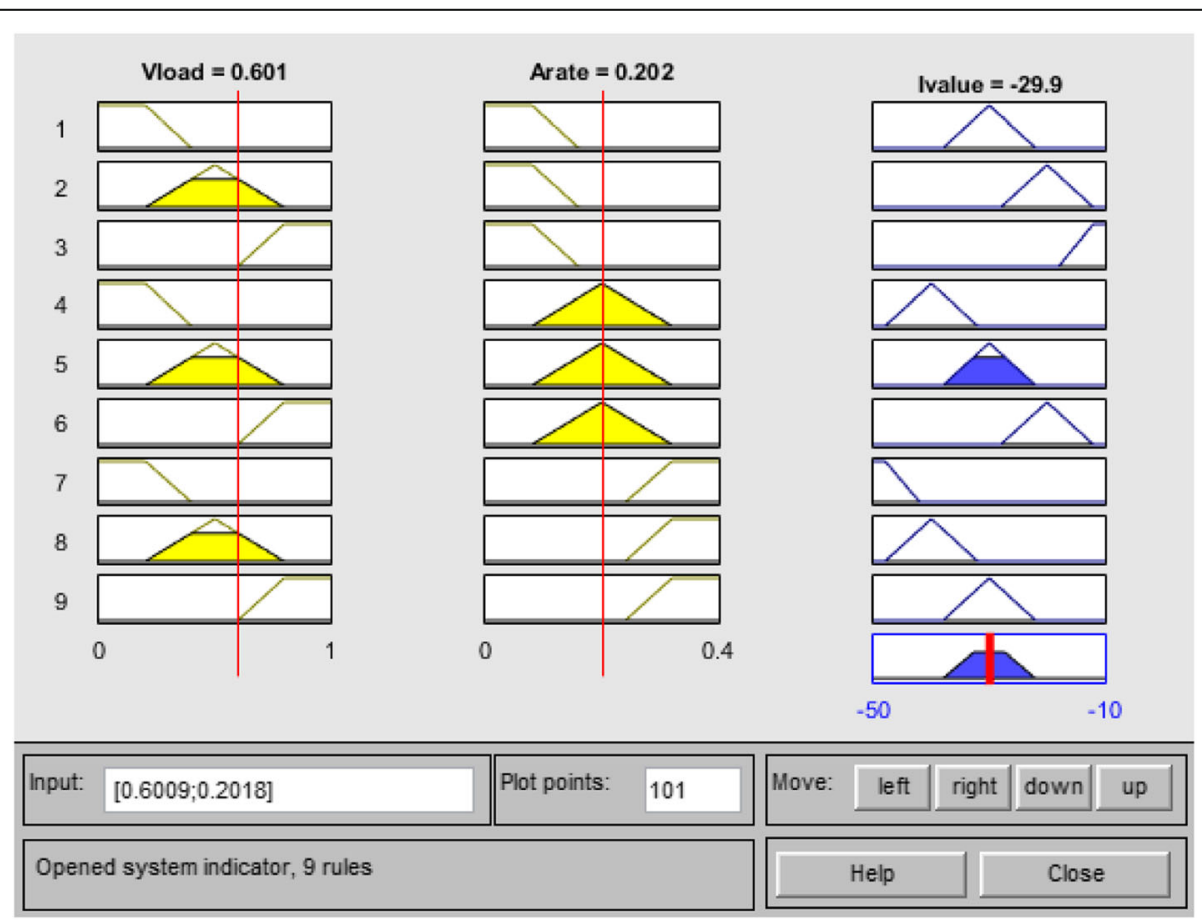

Fig. 5 Value of activation bias factor 
Table 2 Simulation parameters

\begin{tabular}{lll}
\hline Parameters & Unit & Values \\
\hline Operating frequency & $\mathrm{MHz}$ & 3500 \\
Bandwidth & $\mathrm{MHz}$ & 20 \\
BSs density $\left(\lambda_{\mathrm{BS}}\right)$ & $/ \mathrm{km}^{2}$ & $14 / 87 / 174 / 261$ \\
$N_{\text {pilot }}$ & & $40 / 92$ \\
Path loss exponent & & $3 / 4 / 5$ \\
Transmit signal-to-noise rate $(\eta)$ & $\mathrm{dB}$ & 162 \\
Area of simulation & $\mathrm{m}$ & $1000^{*} 1000$ \\
\hline
\end{tabular}

allocation. Thus, we balance data rates and resource overhead by different activation strategies. The preference of activation strategy can be modeled by a utility function.

\subsection{A utility function}

The user receives a non-coherent sum of multiple copies of the useful signal transmitted by active BSs in its own virtual cell. Let $W_{1}$ be the utility value for one bit of transmission, and $W_{2}$ be the cost for one resource block. We define a utility function $Q\left(T, \mathrm{ia}_{x}\right)$ to comprehensively reflect the impact of rates and resources on users. The optimal activation strategy has the largest utility value.

$$
Q\left(T, \mathrm{ia}_{x}\right)=W_{1} C_{T, \mathrm{ia}_{x}}-W_{2} \text { Resource }_{T, \mathrm{ia}_{x}}
$$

But it is difficult to set the specific values of $W_{1}$ and $W_{2}$. We can only empirically give the relative importance of resource and data rates according conditions. Thus, we set $\beta=\frac{W_{2}}{W_{1}}$ as a bias factor of activation strategy. It indicates the relative importance of resource to data rates under different loads and QoS requirements. When $\beta$ is large, the resource is more important than data rates. Conversely, the data rate is more important.

For $\beta$, we can only empirically set its value. It is not enough for solving the utility function. We need to adjust values for different degrees of load and rate. And fuzzy logic technology seems suitable to handle the imprecision problems of the practical wireless network [29]. Fuzzy sets maps the set elements to a membership function with indicates the degree of truth belonging to the set. This helps us to express the imprecision and vagueness in the real networks which cannot be easily studied [30]. Thus, we propose a method based on fuzzy logic theory to get $\beta$.

\subsection{A fuzzy logic-based method to obtain $\beta$}

According to our previous analysis, there are two main influence factors for $\beta$. One is average loads of virtual cells, which reflects the degree of resource shortage. The other is adjustable data rates, which indicates users' requirements. These two factors are set as inputs for the fuzzy logic system (FLS), and the values can be calculated as (14) and (15).

The NCU construct virtual cells with this radius $D_{\text {best }}$ for users. The number of BSs is $N$ in $j$-th virtual cell. It collects the resources usage of BSs in

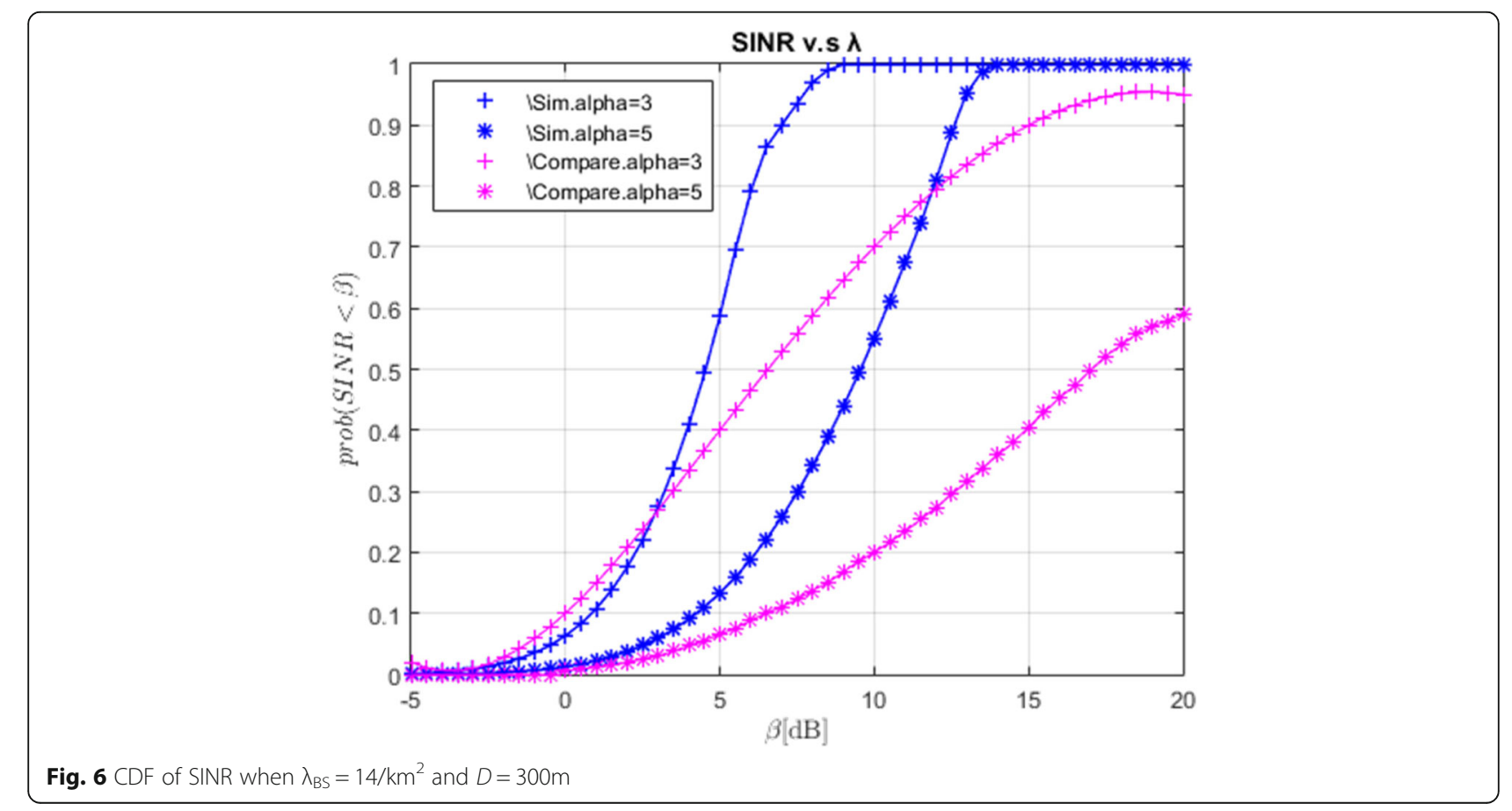




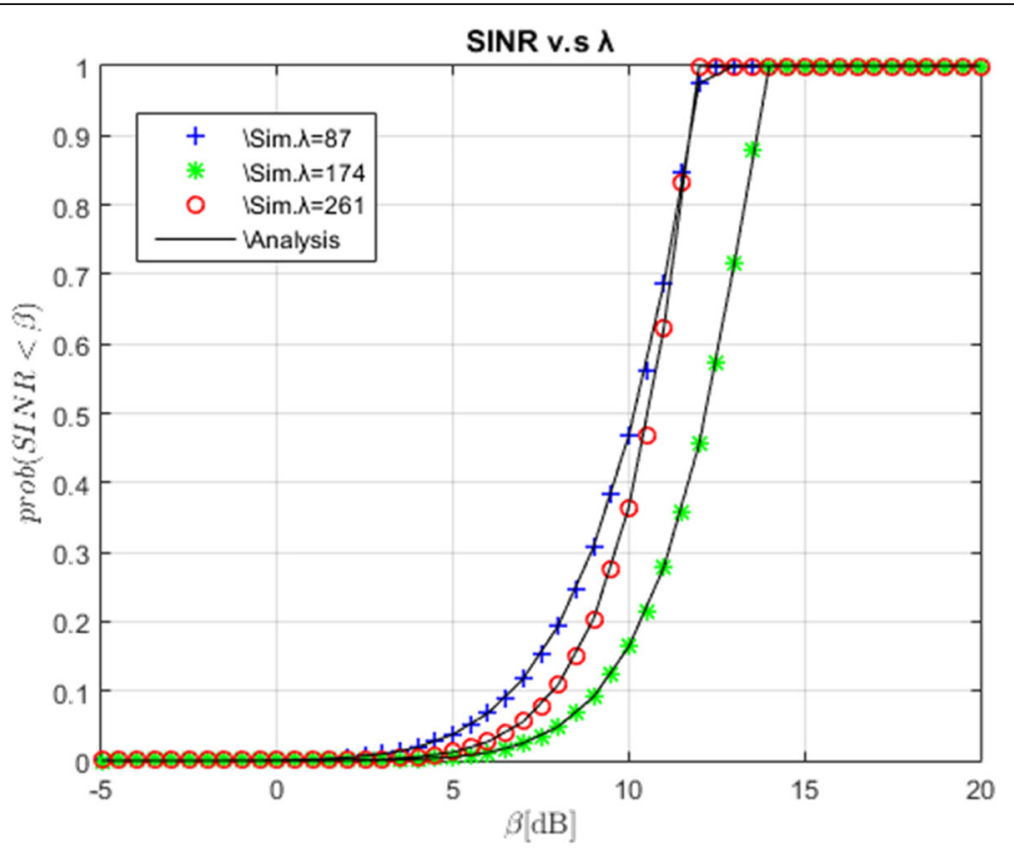

Fig. 7 CDF of SINR when $D=105 \mathrm{~m}$

virtual cell region and calculates the average loads of the virtual cells.

$$
\operatorname{Load}_{V_{j}}(t)=\frac{\sum_{i=1}^{N} N_{i \in V_{j}}}{N \times N_{\text {total }}}
$$

Where, $N_{\text {total }}$ is the total number of physical resource blocks (PRBs) owned by each BS. $N_{i \in V_{j}}$ is the number of PRBs that BS $i$ has consumed at time $t$. BS $i$ is located in $j$-th virtual cell.

The ratio of adjustable data rates is expressed by (15). It indicates users' tolerate ability for data rates change.

$$
\Delta C_{V_{j}}=1-\frac{C_{\mathrm{gbr}}}{C_{\mathrm{mbr}}}
$$

Where, $C_{\mathrm{mbr}}$ and $C_{\mathrm{gbr}}$ is the maximum and guaranteed bit rates of a GBR bearer for downlink.

Our FLS includes two inputs and one output as Fig. 2. We take the results of (14) and (15) as inputs and the value of activation bias factor $\beta$ is the output. Mamdani FIS is used as it is known to be well suited to capture expert knowledge [31]. We adopt "maximum-minimum" fuzzy inference synthesis rule. The defuzzification method is centroid.

The implement of FLS mainly includes four steps:

Step 1: Fuzzification of inputs. There are two inputs, namely, the loads of virtual cells and the ratio of adjustable data rates. Their range both are $0 \sim 1$. The corresponding input fuzzy sets are denoted by $\widetilde{\text { Vload }}$ and $\widetilde{\text { Arate }}$. Each input fuzzy set has three membership functions (low, medium, high). These membership functions setting rely on expert experience. We use triangular and trapezoidal functions to fuzzy inputs. The fuzzy membership functions (FMS) of inputs are shown in Fig. 3.

Step 2: Fuzzification of output. There is an only one output, namely, activation bias factor $\beta$, and its range is $[-50 \mathrm{~dB},-10 \mathrm{~dB}]$. We set output fuzzy set as Ivalue, which contains five fuzzy membership functions (low, medium-low, medium, medium-high, high). The FMS of output is shown in Fig. 4.

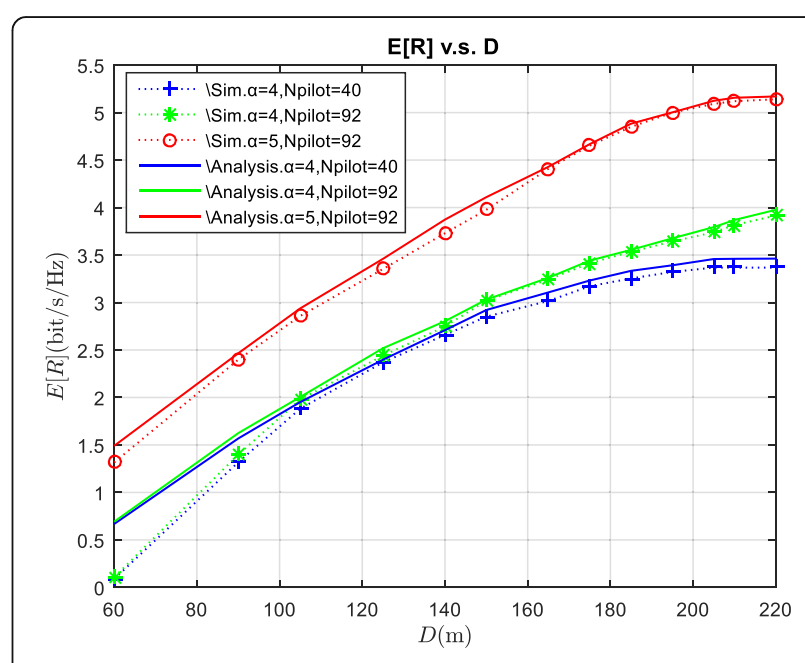

Fig. $8 E[R]$ v.S. $D$ when $\lambda_{B S}=87 / \mathrm{km}^{2}$ 


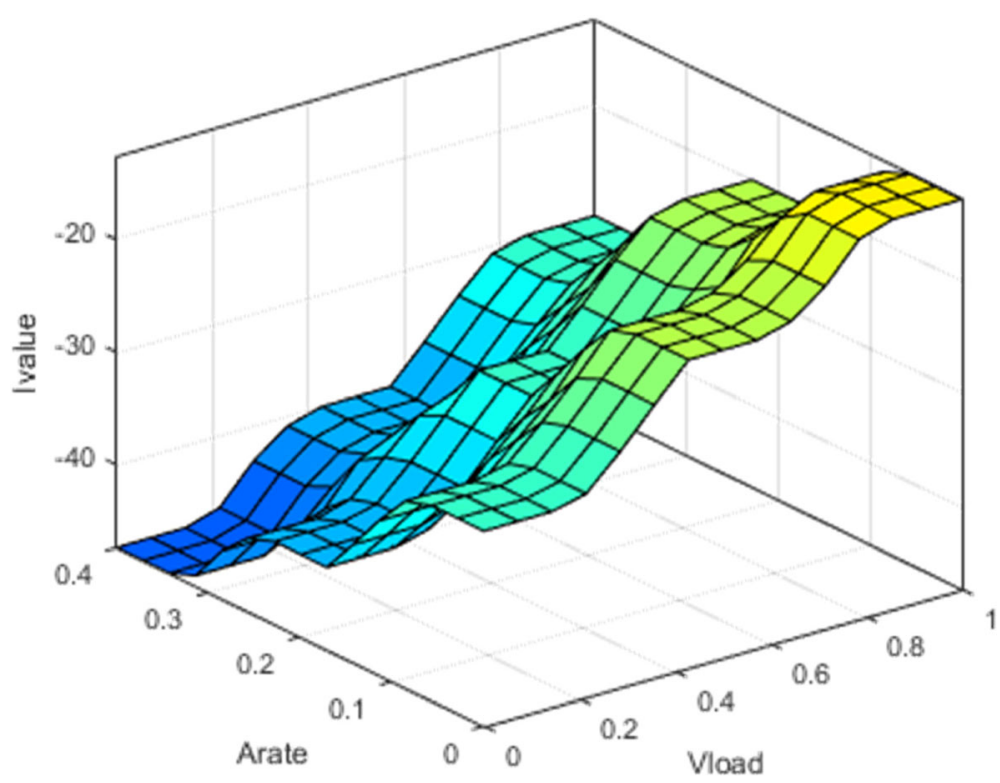

Fig. 9 Surface viewer of activation bias factor

Step 3: Fuzzy reasoning rules. The number of if-then rules is 9 based on Eq. 3 from [32]. Each fuzzy rule is assigned a decision output, which is also based on expert knowledge. The input values during fuzzification process can belong to more than one fuzzy set with the different degreed of membership. Hence, it might trigger several if-then rules as a result. The specific reasoning rules as shown in Table 1. In our FLS, the 9 inference rules in the table below have the same importance, so their weights are all set to 1 .

Step 4: Carry out defuzzification. The aggregated fuzzified Ivalue is given by (Eq. 6 from [32])

$$
\mu \widetilde{\text { Ivalue }}=\max _{k}\left[\min \left[\mu \widetilde{\operatorname{Vload}^{k}}{ }^{k}(\text { load }), \mu \widetilde{\text { Arate }^{k}}\right]\right]
$$

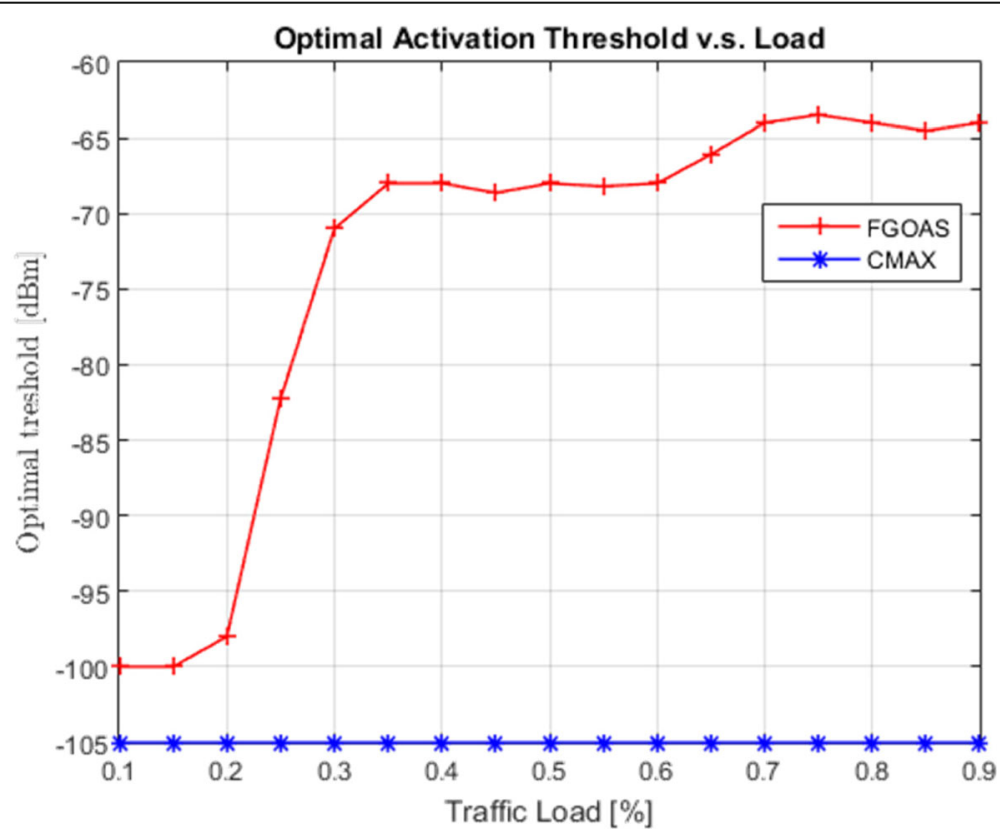

Fig. 10 Optimal activation threshold v.s. traffic load when $\lambda_{B S}=174 / \mathrm{km}^{2} D=135, a=4, N_{\text {pilot }}=92$, Arate $=0.2$, $i a_{x}=i a_{2}$ 
The defuzzifier coverts the aggregated fuzzified value to a crisp value. It is calculated using centroid method (Eq. 6 from [32]):

$$
\text { Ivalue }=\frac{\int \mu \operatorname{Ivalue}(\mathrm{y}) \mathrm{ydy}}{\int \mu \operatorname{Ivalue}(\mathrm{y}) \mathrm{dy}}
$$

Through the above steps, we can get the value of $\beta$ under different loads and QoS conditions as Fig. 5.

\subsection{The optimal activation strategy}

In Section 4.1, we build a utility function for users synthetically consider the effects of loads and rates. In Section 4.2, we get the value of $\beta$ based on the fuzzy logic theory. In this subsection, we can choose the optimal activation strategy to maximize the utility function. It is worth noting that some rules must be observed in selection.

- Only one resource allocated scheme in a virtual cell.

- The number of PRBs that allocated to users cannot exceed bandwidth resources of BSs.

- In order to reach the QoS requirement of users, we have to activate the minimum number of BSs which is at least 1 , even the traffic load is high.

From (13), we know the utility value $Q\left(T, \mathrm{ia}_{x}\right)$ is a function of activation strategy $\left(T\right.$ and $\left.\mathrm{ia}_{x}\right)$. Given the activation bias factor $\beta\left(\right.$ e.g., $\left.W_{2} / W_{1}\right)$, we can find the optimal activation strategy $\left(T, \mathrm{ia}_{x}\right)^{*}$.

$$
\begin{gathered}
\left(T, \mathrm{ia}_{x}\right)^{*}=\arg \max Q\left(T, \mathrm{ia}_{x}\right) \\
\text { s.t. } C 1: \mathrm{ia}_{x}=\left\{\mathrm{ia}_{1}, \mathrm{ia}_{2}\right\} \\
C 2: N_{\mathrm{i} \in V_{j}}<N_{\text {total }} \\
C 3: K \geq 1
\end{gathered}
$$

We calculate the utility values for all possible activation strategies (e.g., $T$ and $\mathrm{ia}_{x}$ ). In the calculation process, we set a comparison baseline for reducing computational complexity. When activation threshold $T=0$, the activation indictor $\mathrm{ia}_{x}$ has no effect on the system spectral efficiency. In this case, the BSs in a virtual cell will all be activated, and the spectral efficiency of the virtual cell will reach the data rates upper limit $C_{\mathrm{mbr}}$ and resource consumption of NCJT

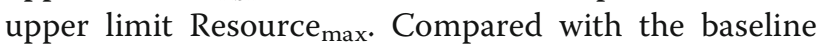
case, the normalized value of data rates and resource can be calculated as (19) and (20).

$$
\begin{aligned}
& N C_{T, \mathbf{i}_{x}}=\frac{C_{T, \mathbf{i}_{x}}}{C_{\mathrm{mbr}}} \\
& \text { NResource }_{T, \mathrm{i}_{x}}=1-\triangle \text { Resource }
\end{aligned}
$$

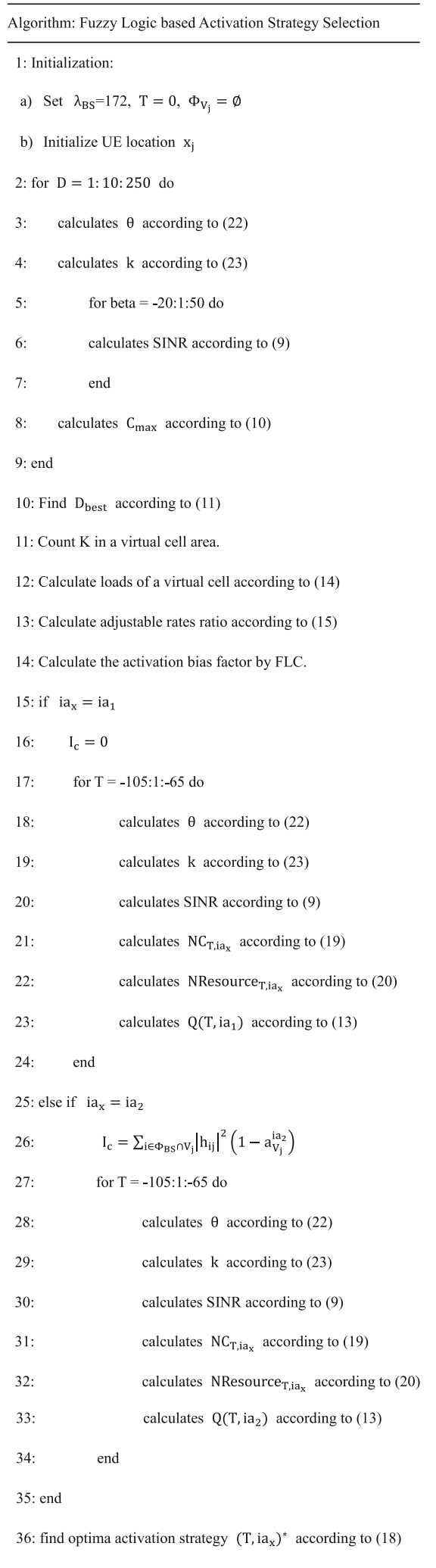

The specific algorithm is as follows: 


\section{Results}

Monte Carlo simulations are carried out to evaluate the performance of proposed virtual cell design and the FAAS algorithm in UDN environment. Simulation parameters are in the Table 2.

\subsection{Evaluation impact of the imperfect CSI}

In our virtual cell design, the effects of imperfect CSI channels are considered. We compare the SINR of our proposed virtual cell design with the virtual cell based on perfect CSI [26]. In Fig. 6, we can see that imperfect CSI can significantly affect users' SINR and course approximately $10 \mathrm{~dB}$ decrease. Thus, the impact of imperfect CSI cannot be ignored.

\subsection{Evaluation the optimal radius of virtual cell}

Figure 7 compares the SINR from (9) with the empirical CDF obtained through simulation. From our previous analysis, we know that the aggregate interference includes three parts and it is approximated by a gamma random variable. From Fig. 7, we can see this approximation matches the empirical SINR very well. With $\lambda_{\mathrm{BS}}$ increases, the distances between users and their potential service BSs reduce. It gives BSs more chances to cooperate. But it does not mean that the density can be increased indefinitely. In Fig. 7 , the SINR of $\lambda_{\mathrm{BS}}=174 / \mathrm{km}^{2}$ is better than $\lambda_{\mathrm{BS}}=261 / \mathrm{km}^{2}$. This is because of interference sources that also increase with the $\lambda_{\mathrm{BS}}$. The residual interference due to imperfect CSI will increase until offset most signal gain. There is an optimal $\lambda_{\mathrm{BS}}$ to maximize the SINR.

Thus, we know that there is an optimal radius for virtual cell when the $\lambda_{\mathrm{BS}}$ is constant. Furthermore, considering the constriction of resources, the radius $D$ should not be too large. This trend can be seen in Fig. 8. We can find the saturate point is around $D=$ 185 when $\alpha=4$ and $N_{\text {pilot }=92}$. From previous analysis, we also know the system average spectral efficiency is influenced not only by the virtual cell radius but also by path loss exponent and the pilot resources blocks. In Fig. 8, we also see that the spectral efficiency saturation point increase with $\alpha$ and $N_{\text {pilot }}$.

\subsection{Evaluation the optimal activation strategy}

We can comprehensively calculate the impact of different traffic loads and adjustable rates ratio on $\beta$ by the fuzzy logic system. The result is as Fig. 9 and the unit of value is decibel. When it is above 0 , it means that the importance of resources is higher than data rates. In most cases, the value of factor is below 0 . It indicates our main pursuit to increase rate. The value of factor increase gently with the loads and adjustable rates. When a user experiences a high-load condition and adjustable rates ratio is small, it will change the first task to save resources.

The optimal activation threshold $T$ by our design can be seen in Fig. 10. The adjustable rate of the virtual cell is set to $20 \%$ of $C_{\mathrm{mbr}}$. The optimal activation threshold $T$ gradually increases with traffic loads. In light-load condition, virtual cells prefer to aggressively active their all BSs in order to maximize data rates. In medium or high loads, virtual cells prefer to activate several BSs with high RSRP to obtain the balance between data rates and resource overhead. When resources allocated scheme indicator are $\mathrm{ia}_{1}$, activation threshold can change the number of activated BSs in a virtual cell and affect the data rates. However, it is noteworthy that the activation threshold will not affect the proportion of resource saving. In this case, the use of resources is the same as that of the baseline case. There are no resources reduced. The simulation results show that the proposed algorithm dynamically adjust activation threshold according to different traffic loads conditions and improve the adaptability of virtual cell in practical scenario.

\section{Conclusions}

In this paper, we analyzed the challenges of ultradense network in 5G. UDN becomes a pillar technology for the $5 \mathrm{G}$ cellular networks. In order to harvest densification gains, we proposed a virtual cell design to overcome the complex interference and frequent handover.

In our article, we adopt the NCJT as the enabler technology to construct the user-centric virtual cells. However, it consumes more resources and has a limitation in using. For breaking this constriction, we need to rationally choose activation strategies for virtual cells to balance data rates and resource allocation. We consider the practical scenario of UDN and use random geometry tools to model network with the imperfect CSI. We find the optimal virtual cell radius to maximize the average system spectral efficiency. On this basis, we propose a FASS algorithm to find the optima activation strategy. Users can achieve higher system spectral efficiency, better service experience, and seamless moving.

However, NCJT is not the optimal transmission technology when some users are close to each other. Multiuser cooperative transmission could bring performance improvement in high-loaded areas instead of sacrificing performance to ensure resource utilization. This will be discussed in our future work. 


\section{Appendix}

$$
\begin{aligned}
& E\left[e^{-s R}\right]=E\left[e^{-s\left(1-\sigma_{\mathrm{MMSE}, i j}{ }^{2}\right)\left|h_{i j}\right|^{2} a_{V_{j}}^{i}}\right]^{K} \\
& =E\left[\int_{0}^{D} \frac{2 r}{D^{2}} e^{-s\left(1-\sigma_{\mathrm{MMSE}, i j^{2}}\right) f_{i j}{ }^{2} r^{-\alpha} a_{V j}^{i}} d r\right]^{K} \\
& =E\left[\frac{2}{\alpha D^{2}} \int_{D^{-\alpha}}^{\infty} t^{-1-\frac{2}{\alpha}} e^{-s\left(1-\sigma_{\mathrm{MMSE}, i j^{2}}\right) f_{i j}{ }^{2} t a_{V_{j}}^{i}} d t\right]^{K} \\
& =E\left[\frac{2}{\alpha D^{2}} \int_{D^{-\alpha}}^{\infty} t^{-1-\frac{2}{\alpha}} e^{-s\left(1-\sigma_{\mathrm{MMSE}, i j}{ }^{2}\right) f_{i j}^{2} t t>T / f_{i j}^{2}} d t\right]^{\mathrm{K}} \\
& =\left(\frac{2}{\alpha D^{2}} \int_{D^{-\alpha}}^{\infty} t^{-1-\frac{2}{\alpha}}\left(1-e^{-\frac{T}{t}}+\frac{e^{-\frac{T}{t}(u+1)}}{u+1}\right) d t\right)^{\mathrm{K}}
\end{aligned}
$$

Where, $u=\frac{s t^{2}}{\mathrm{t}+K /\left[N_{\text {pilot }}\left[\left(\left[\bar{C}^{3}\right)+1 / \eta\right]\right.\right.}$

$$
\begin{aligned}
& \theta=\frac{\frac{\pi \lambda}{\alpha-1} E\left[f_{i j}{ }^{4} \min \left\{D^{\alpha}, \frac{T}{f_{i j}{ }^{2}}\right\}^{\frac{2}{\alpha}-2}\right]+\frac{K}{b^{2} D} E\left[f_{i j}{ }^{4} \min \left\{D^{\alpha}, \frac{T}{f_{i j}{ }^{2}}\right\}^{\frac{2}{\alpha}-2} \times{ }_{2} F_{1}\left(2, \frac{2}{\alpha}, 1+\frac{2}{\alpha} ;-b^{-1} \min \left\{D^{\alpha}, \frac{T}{f_{i j}{ }^{2}}\right\}\right)\right]-\frac{K}{b^{2} D^{4}} E\left[f_{i j}{ }^{2} \min \left\{D^{\alpha}, \frac{T}{f_{i j}{ }^{2}}\right\}^{\frac{2}{\alpha}} \times{ }_{2} F_{1}\left(1, \frac{2}{\alpha}, 1+\frac{2}{\alpha} ;-b^{-1} \min \left\{D^{\alpha}, \frac{T}{f_{i j}{ }^{2}}\right\}\right)\right]}{\frac{2 \pi \lambda}{\alpha-2} E\left[f_{i j}{ }^{2} \min \left\{D^{\alpha}, \frac{T}{f_{i j}{ }^{2}}\right\}^{\frac{2}{\alpha}-1}\right]+\frac{1}{\eta}+\frac{K}{b D^{2}} E\left[f_{i j}{ }^{2} \min \left\{D^{\alpha}, \frac{T}{f_{i j}{ }^{2}}\right\}^{\frac{2}{\alpha}} \times{ }_{2} F_{1}\left(1, \frac{2}{\alpha}, 1+\frac{2}{\alpha} ;-b^{-1} \min \left\{D^{\alpha}, \frac{T}{f_{i j}{ }^{2}}\right\}\right)\right]}
\end{aligned}
$$

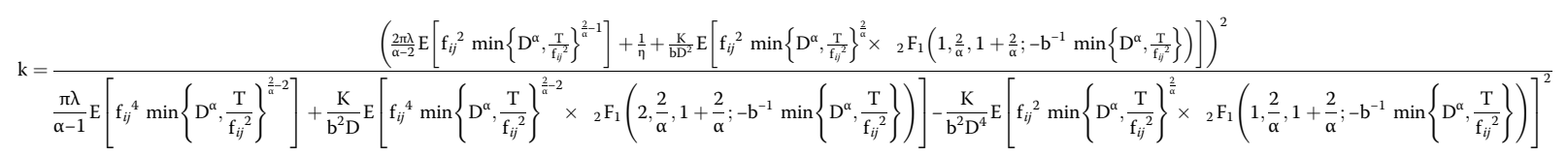

\section{Abbreviations}

5G: The fifth generation cellular network; CoMP: Coordinated multipoint transmission; C-plan: Control plane; CSI: Channel state information; DAS: Distributed antenna system; FASS: Fuzzy logic-based activation strategy selection; FLS: Fuzzy logic system; MIMO: Virtual multiple-input multipleoutput; NCJT: Non-coherent joint transmission; NCU: Network controller unit; QoS: Quality of service; UDN: Ultra-dense networks; U-plan: User plan

\section{Funding}

This work described in this paper was supported by Ministry of Science and Technology of the People's Republic of China (Grant No. 2018ZX03001029-004).

\section{Authors' contributions}

QL conceived the study and performed the simulation experiments. KSZ wrote the paper. WDG and GC reviewed and edited the manuscript. All authors read and approved the final manuscript.

\section{Authors' information}

Qian Liu is currently working toward the Ph.D. degree with the School of Information and Communication Engineering, Beijing University of Posts and Telecommunications.

\section{Competing interests}

The authors declare that they have no competing interests.

\section{Publisher's Note}

Springer Nature remains neutral with regard to jurisdictional claims in published maps and institutional affiliations.

Received: 11 February 2018 Accepted: 11 April 2018

Published online: 25 April 2018

References

1. A Gotsis, S Stefanatos, A Alexiou, Ultradense networks: the new wireless frontier for enabling $5 G$ access. IEEE Veh. Technol. Mag. 11(2), 71-78 (2016) 
2. M Kamel, W Hamouda, A Youssef, Ultra-dense networks: a survey. IEEE Commun. Surv. Tutorials 18(4), 2522-2545 (2016)

3. LM Loew, JC Schaff, The Virtual Cell: a software environment for computational cell biology[]]. TRENDS in Biotechnology 19(10), 401-406 (2001)

4. S Chen, F Qin, B Hu, X Li, Z Chen, User-centric ultra-dense networks for 5G: challenges, methodologies, and directions. IEEE Wirel. Commun. 23(2), 78-85 (2016)

5. A Beylerian, T Ohtsuki, Service-aware user-centric clustering and scheduling for cloud-RAN with coordinated multi-point transmission. In Signal and Information Processing Association Annual Summit and Conference (APSIPA), 2015 Asia-Pacific (pp. 252-257). IEEE

6. V Garcia, Y Zhou, J Shi, Coordinated multipoint transmission in dense cellular networks with user-centric adaptive clustering. IEEE Trans. Wirel. Commun. 13(8), 4297-4308 (2014)

7. Y Liu, X Li, H Ji, K Wang, H Zhang, Joint APs selection and resource allocation for self-healing in ultra dense network. In Computer, Information and Telecommunication Systems (CITS), 2016 International Conference on (pp. 1-5). IEEE

8. L Liu, V Garcia, L Tian, Z Pan, J Shi, Joint clustering and inter-cell resource allocation for CoMP in ultra dense cellular networks. In Communications (ICC), 2015 IEEE International Conference on (pp. 2560-2564). IEEE

9. F Zhao, L Wei, H Chen, Optimal time allocation for wireless information and power transfer in wireless powered communication systems. IEEE Trans. Veh. Technol. 65(3), 1830-1835 (2016)

10. J Li, A Papadogiannis, R Apelfröjd, T Svensson, M Sternad, Performance evaluation of coordinated multi-point transmission schemes with predicted CSI. In Personal Indoor and Mobile Radio Communications (PIMRC), 2012 IEEE 23rd International Symposium on (pp. 1055-1060). IEEE

11. X Li, H Xia, Z Zeng, A novel user-centric comp scheme for energy efficiency of dense heterogeneous network. In Network Infrastructure and Digital Content (IC-NIDC), 2016 IEEE International Conference on (pp. 145-149). IEEE

12. L Liu, Y Zhou, V Garcia, L Tian, J Shi, Load Aware Joint CoMP Clustering and Inter-Cell Resource Scheduling in Heterogeneous Ultra Dense Cellular Networks. IEEE Trans. Veh. Technol. 67(3), 2741-2755

13. T Kanakis, MO Agyeman, A Bakaoukas, Adaptive virtual MIMO single cluster optimization in a small cell. In Cloud Computing, Data Science \& EngineeringConfluence, 2017 7th International Conference on (pp. 474-478). IEEE

14. HL Kim, S Chong, Virtual cell beamforming in cooperative networks. IEEE J. Selected Areas Commun. 32(6), 1126-1138 (2014)

15. B Kwon, S Lee, Effective interference nulling virtual MIMO broadcasting transceiver for multiple relaying. IEEE Access 5, 20695-20706 (2017)

16. A Barbieri, P Gaal, S Geirhofer, T Ji, D Malladi, Y Wei, F Xue, Coordinated downlink multi-point communications in heterogeneous cellular networks. In Information Theory and Applications Workshop (ITA), 2012 (pp. 7-16). IEEE

17. R Tanbourgi, S Singh, JG Andrews, FK Jondral, Analysis of non-coherent joint-transmission cooperation in heterogeneous cellular networks. In Communications (ICC), 2014 IEEE International Conference on (pp. 5160-5165). IEEE

18. J Park, SL Kim, J Zander, Asymptotic behavior of ultra-dense cellular networks and its economic impact. In Global Communications Conference (GLOBECOM), 2014 IEEE (pp. 4941-4946). IEEE

19. D Liu et al., User association in $5 \mathrm{G}$ networks: a survey and an outlook. IEEE Commun. Surv. Tutorials 18(2), 1018-1044 (2016)

20. M Ding, D López-Pérez, G Mao, P Wang, Z Lin, Will the area spectral efficiency monotonically grow as small cells go dense?. In Global Communications Conference (GLOBECOM), 2015 IEEE (pp. 1-7). IEEE

21. H ElSawy, E Hossain, M Haenggi, Stochastic geometry for modeling, analysis, and design of multi-tier and cognitive cellular wireless networks: a survey. IEEE Commun. Surv. Tutorials 15(3), 996-1019 (2013)

22. H Ibrahim et al., Mobility-aware modeling and analysis of dense cellular networks with C-plane/U-plane split architecture. IEEE Trans. Commun. 64(11), 4879-4894 (2016)

23. A Lapidoth, S Shamai, Fading channels: how perfect need "perfect side information" be? IEEE Trans. Inf. Theory 48(5), 1118-1134 (2002)

24. B Hassibi, BM Hochwald, How much training is needed in multiple-antenna wireless links? IEEE Trans. Inf. Theory 49(4), 951-963 (2003)

25. M Haenggi, RK Ganti, Interference in large wireless networks. Foundations and Trends ${ }^{\circledast}$ in Networking 3(2), 127-248 (2009)

26. R Tanbourgi et al., A tractable model for noncoherent joint-transmission base station cooperation. IEEE Trans. Wirel. Commun. 13(9), 4959-4973 (2014)
27. RW Heath, M Kountouris, T Bai, Modeling heterogeneous network interference using Poisson point processes. IEEE Trans. Signal Process. 61(16), 4114-4126 (2013)

28. S Singh, HS Dhillon, JG Andrews, Offloading in heterogeneous networks: Modeling, analysis, and design insights. IEEE Trans. Wirel. Commun. 12(5), 2484-2497 (2013)

29. JM Mendel, Uncertain rule-based fuzzy logic systems: introduction and new directions (p. 4). (Prentice Hall PTR, Upper Saddle River, 2001)

30. G Klir, B Yuan, Fuzzy sets and fuzzy logic (Vol. 4). (Prentice hall, New Jersey, 1995)

31. T Thumthawatworn, Adaptive membership functions for handover decision system in wireless mobile network. Procedia Comput. Sci. 86, 31-34 (2016)

32. T Thumthawatworn, A Pervez, P Santiprabhob, Adaptive modular fuzzybased handover decision system for heterogeneous wireless networks. Int. J. Netw. Commun. 3(1), 25-32 (2013)

\section{Submit your manuscript to a SpringerOpen ${ }^{\circ}$ journal and benefit from:}

- Convenient online submission

- Rigorous peer review

- Open access: articles freely available online

- High visibility within the field

- Retaining the copyright to your article

Submit your next manuscript at $>$ springeropen.com 